\title{
ResearchOnline@JCU
}

This is the Accepted Version of a paper published in the journal: Nano Letters

Jacob, Mohan V., Rawat, Rajdeep S., Ouyang, Bo, Bazaka, Kateryna, Kumar,

D. Sakthi, Taguchi, Dai, Iwamoto, Mitsumasa, Neupane, Ram, and Varghese, Oomman K. (2015) Catalyst-free plasma enhanced growth of graphene from sustainable sources. Nano Letters, 15. pp. 5702-5708.

http://dx.doi.org/10.1021/acs.nanolett.5b01363

(C) 2015. This manuscript version is made available under the CC-BY-NC-ND 4.0 license

http://creativecommons.org/licenses/by-nc-nd/4.0/

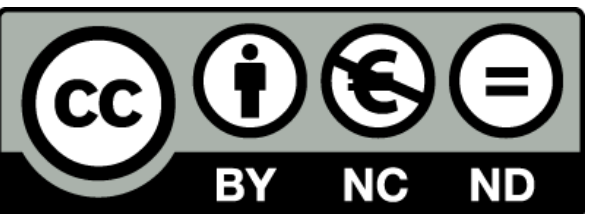




\section{Catalyst free plasma enhanced growth of graphene from sustainable sources}

Mohan V. Jacob', Rajdeep S. Rawat ${ }^{2}$, Bo Ouyang², Kateryna Bazaka1, D. Sakthi Kumar ${ }^{3}$, Dai Taguchi ${ }^{4}$, Mitsumasa Iwamoto ${ }^{4}$, Ram Neupane ${ }^{5}$ and Oomman K. Varghese $^{5}$

${ }^{1}$ Electronics Materials Lab, College of Science, Technology and Engineering, James Cook University, Townsville, QLD 4811 Australia

${ }^{2}$ Natural Sciences and Science Education, National Institute of Education, Nanyang Technological University, 637616 Singapore

${ }^{3}$ Bio Nano Electronics Research Center, Graduate School of Interdisciplinary New Science Toyo University, Kawagoe, Saitama, 350 - 8585, Japan

${ }^{4}$ Department of Physical Electronics, Tokyo Institute of Technology, O-okayama, Meguro-ku, Tokyo 152-8552, Japan

${ }^{5}$ Nanomaterials and Devices Laboratory, Department of Physics, University of Houston, Houston, Texas 77204, USA

\section{Abstract:}

Details of a fast and sustainable bottom up process to grow large area high quality graphene films without the aid of any catalyst are reported in this paper. We used Melaleuca alternifolia, a volatile natural extract from tea tree plant as the precursor. The as-fabricated graphene films yielded a stable contact angle of $135^{\circ}$, indicating their potential application in very high hydrophobic coatings. The electronic devices formed by sandwiching pentacene between graphene and aluminum films demonstrated memristive behavior and hence, these graphene films could find use in non-volatile memory devices also.
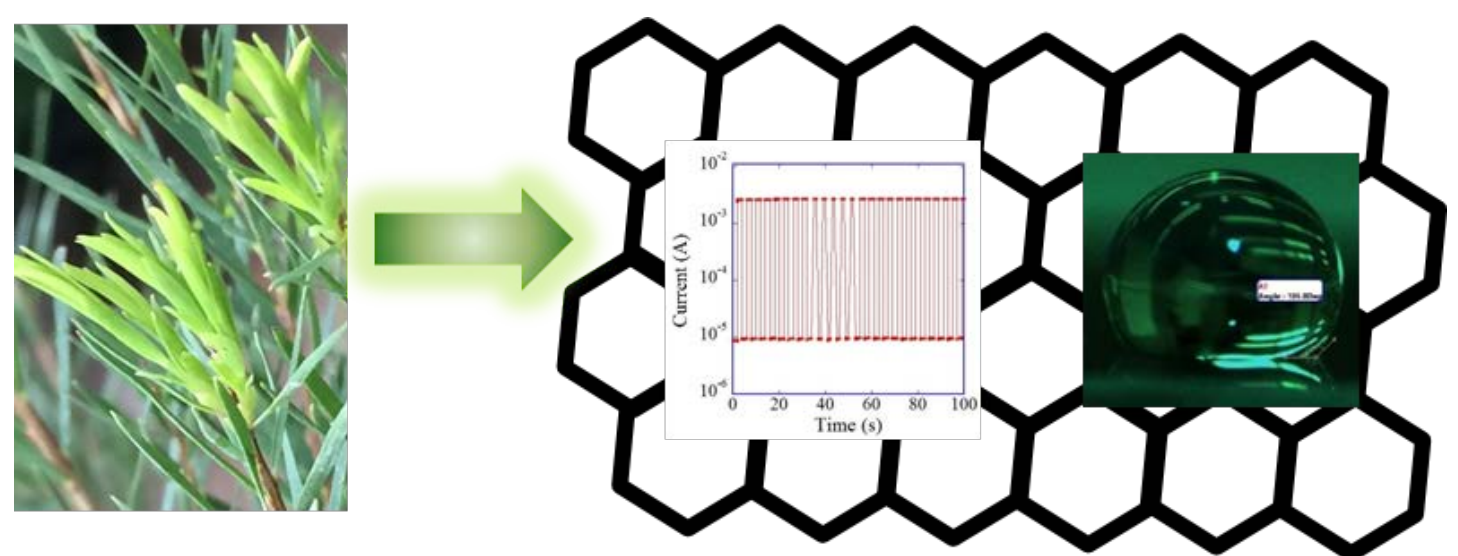

TOC Graph: Fabrication of graphene using PE-CVD from agricultural based volatile precursor 
Graphene is featured by a rare combination of unique properties highly sought after for a wide spectrum of applications particularly in areas such as electronics, photonics, energy and environmental sensing. Monolayer hexagonal lattice that is impermeable to even helium, band gap tunability in the range 0 to $\sim 0.3 \mathrm{eV}$, charge carriers that can be described as massless Dirac Fermions and ballistic transport of carriers with room temperature electron mobility exceeding $15000 \mathrm{~cm}^{2} / \mathrm{Vs}$ (predicted value $>10^{5} \mathrm{~cm}^{2} / \mathrm{Vs}$ ) even at low carrier concentration are some of the distinct characteristics of graphene ${ }^{1-4}$. These properties along with high transmittance in the visible region of the solar spectrum, high mechanical robustness and high thermal conductivity make graphene attractive for transparent electrode applications, high strength light weight composites, micro- and nano-mechanical systems, flexible and printable optoelectronics, thin film transistors (TFTs) and photonic devices ${ }^{5}$. Graphene is also highly chemically reactive along its edges and defects, demonstrating reliable responses to chemical and biologically-relevant species ${ }^{6}$.

Graphene emerged as a material of high scientific and technological relevance shortly after the first practical illustration of its isolation from graphite ${ }^{5}$. Nevertheless, large scale use of graphene has not yet been realized. Scarcity of scalable, environmentally sustainable and economically viable processes for producing device quality graphene has been generally recognized as an issue.

Development of appropriate technologies to grow device quality graphene films over sufficiently large area, depending upon the application, is crucial for the realization of graphene based commercial devices. The technology must be sustainable, scalable and economically feasible. Although mechanical exfoliation from graphite crystals is one of the simplest methods originally explored to obtain graphene, it is not suitable to yield large area films. Bottom-up strategies like epitaxial growth and chemical vapor deposition (CVD) are appropriate for mono or multilayer coatings of graphene over large areas. A majority of currently available methods, including epitaxial growth and CVD, rely on complex, energy intensive processes that employ toxic or hazardous precursors and require copper or nickel substrates for growing high quality graphene ${ }^{7}$. For example, a typical CVD process for the growth of graphene requires a temperature exceeding $1000{ }^{\circ} \mathrm{C}$, growth duration of several hours and methane mixed with hydrogen as precursor. 
Plasma enhanced CVD (PECVD) has been employed to reduce the process temperature for graphene deposition. Plasma environment is effective in breaking the bonds of organic precursors including polymers and reform and organize carbon nanomaterials to graphene or carbon nanotubes. Plasma generated by radio frequency (rf), direct current (dc) or microwave power is generally used. Yang et al grew single domain graphene at $500{ }^{\circ} \mathrm{C}$ using methane plasma followed by etching of graphene in hydrogen plasma ${ }^{8}$. Nevertheless, the growth was substrate specific and process duration was about $3 \mathrm{~h}$. Peng et al used PECVD to grow graphene on nickel catalyst using methane with no hydrogen at $475{ }^{\circ} \mathrm{C}{ }^{9}$. The quality of the graphene films obtained was, however, relatively low as indicated by the low intensity of the 2D peak in the Raman spectrum. Catalyst free graphene formation was reported by Kim et al Yang et al and Zhang et al ${ }^{10-13}$ also, but they used $\mathrm{CH}_{4}$ as precursor and fabrication temperature were also relatively high $\left(>1000^{\circ} \mathrm{C}\right)$.

Precursors used for growing graphene have a critical role in deciding the viability of a technology. In CVD based processes, hydrocarbons like methane are primarily used. Generally, the precursors are derived from non-renewable sources and are mostly toxic or explosive. There are reports about using solid polymethylmethacrolyte (PMMA) and polystyrene and liquid benzene for CVD graphene growth ${ }^{14}$. Unless the precursor is derived from renewable sources and are environmental friendly, the process cannot be considered sustainable. In an effort to introduce simple green processes, attempts have been recently made to develop methodologies that can utilize a wide variety of precursors that include food materials such as honey ${ }^{6}$, eggs ${ }^{15}$, cookies ${ }^{16}$ and table sugar ${ }^{6,17}$ natural and plastic waste ${ }^{16}$, and other solid carbon sources ${ }^{18}$. Most of these materials are exotic, the scalability and sustainability are, however, questionable.

Our interest was in developing high quality graphene films with unique properties using a simple sustainable pathway. We developed a radio frequency (rf) capacitively coupled plasma process ${ }^{19-}$ ${ }^{21}$ to dissociate the complex Melaleuca alternifolia vapors at about $800{ }^{\circ} \mathrm{C}$ and form graphene very quickly (seconds to a few minutes) on a variety of substrates including silicon and fused silica without using any catalysts. This extract is an abundant commercial resource obtained by distillation from leaves of M. alternifolia, commonly known as tea-tree. It is hydrocarbon-rich containing over 100 compounds. The primary components are terpinen-4-ol $\left(\mathrm{C}_{10} \mathrm{H}_{18} \mathrm{O}, 30-48 \%\right)$, 
$\gamma$-terpinene $\left(\mathrm{C}_{10} \mathrm{H}_{16}, 10-28 \%\right)$, $\alpha$-terpinene $\left(\mathrm{C}_{10} \mathrm{H}_{16}, 5-13 \%\right)$ and 1,8-cineole $\left(\mathrm{C}_{10} \mathrm{H}_{18} \mathrm{O}, 0-15 \%\right)$. We used a simple growth setup consisting of quartz tube with a heater coil wound around it and two cylindrical rf electrodes placed on either sides of the heater coil. The M. alternifolia extract is volatile, with a flash point of $64{ }^{\circ} \mathrm{C}$ and vapor pressure of 15.7 Torr, and therefore the monomer vapors could be produced at room temperature and fed into the deposition chamber in the same way as conventional sources like methane. We note that the use of a renewable minimally processed monomer, such as M. alternifolia, and a green catalyst-free low temperature plasmaenabled production method is an important step towards environmentally-sustainable fabrication of good quality large area graphene sheets. Detailing this process as well as quality and utility of the fabricated graphene is the goal of this communication.

The graphene films were formed as soon as the plasma was switched on. Figure 1 a-d shows the evolution of graphene morphology with fabrication duration. There is no significant changes in SEM are observed beyond 4 mins. The fast defect-free formation of graphene over a large area is evident from Figures 1, S1 and S2. The images indicate that graphene growth starts in two dimensions (2D) with small protruded features in z-direction. The growth becomes more or less vertical as the deposition time increases. The strain introduced during the fast growth is believed to be primarily responsible for this 3D growth. Figure S3 shows the AFM image of the 2 min sample, which reveals vertically aligned graphene growth with high surface roughness. The folds/edges are prominent for growth time greater than 1 min. Compared to 2D graphene, the vertically oriented graphene has certain specific characteristics such as high surface area and very long edges giving unique functionalities. From the average circumference (about $600 \mathrm{~nm}$ ) of the top edge of a well-like structure shown in Fig. 1c, we estimated the total length of the edges/folds in $1 \mathrm{~cm}^{2}$ area of a sample as $\sim 2.6 \mathrm{~km}^{6}$. Such ultra-long edges together with enhanced surface area make the vertical graphene highly versatile ${ }^{22}$. For instance, the material can be used for developing field emitters and chemical/bio sensors with exceptional performance and highly active electrodes for electrochemical applications.

It can be understood from Transmission electron microscope (TEM) image given in Figure 1e that the graphene films are multilayered. About 3 to 4 layers can be seen in this image, which is of a 2 min sample. The glancing angle x-ray diffraction (GAXRD) pattern (Figure 1f) exhibited 
characteristic hump centered at about $21^{\circ}$ for the 2 min sample, indicating the crystalline nature of this multilayered sample.

The x-ray photoelectron spectroscopy (XPS) studies performed on samples deposited at different durations showed only carbon C1s peak. The typical survey spectrum of sample grown for $2 \mathrm{~min}$ that shows a single C1s peak at about $284 \mathrm{eV}$ is shown in Figure 2a. The spectra from samples prepared under various durations (1s and 1, 2 and 4 min.) looked identical, in general. The fact that no elemental impurity was observed, although the original precursor was multicomponent, shows the high quality of the graphene films obtained from the tea-tree extract.

The high resolution XPS scan of the $284 \mathrm{eV}$ peak in 2 min sample is given in Figures 2b. Similar spectra from other samples are given in Figure S4. The deconvolution of the peak resulted in a major peak at $284.0 \pm 0.2 \mathrm{eV}$ that corresponds to $\mathrm{sp}^{2}$ carbon, two minor peaks at $284.7 \pm 0.1 \mathrm{eV}$ and $285.6 \pm 0.2$ and a $\pi-\pi^{*}$ satellite peak at about $290.5 \pm 0.1 \mathrm{eV}$. The minor peak at $284.7 \mathrm{eV}$ is due to a small amount of $\mathrm{sp}^{3}$ hybridized carbon in the film that might be arising from the structural defects within graphene lattice. Raman studies, discussed later, also gave evidences that support this conclusion. The samples fabricated for the process duration of about 2 min yielded the highest $\mathrm{sp}^{2}$ carbon concentration. The peaks at 285.6 and $290.5 \mathrm{eV}$, which are very small, are assigned to the $\mathrm{C}-\mathrm{O}$ and $\mathrm{O}-\mathrm{C}=\mathrm{O}$ bonds. Oxygen functionalities present in the monomer is believed to be responsible for these bonds.

The high material quality of the graphene was further confirmed using Raman Spectroscopy. Figure 3 shows the Raman spectra of graphene fabricated at different plasma discharge durations. The signature of a single layer of graphene is a sharp second order Raman scattering peak at 2680 $\mathrm{cm}^{-1}$ (2D peak). Decoupled multilayer graphene also exhibits a single intense 2D peak, but its position and the full width at half maximum (FWHM) are affected by the number of layers. Raman spectra of our graphene samples showed a 2D peak at $2695 \mathrm{~cm}^{-1}$, confirming the graphene formation ${ }^{23}$. The samples grown at processing times of $1 \mathrm{~s}, 1$ min and 4 min showed a FWHM of approximately $60 \mathrm{~cm}^{-1}$ at $2695 \mathrm{~cm}^{-1}$, indicating a four layer system. In the case of the graphene produced after two minutes of synthesis, the $2 \mathrm{D}$ peak was observed at $2705 \mathrm{~cm}^{-1}$ and was sharp compared to 2D peaks from samples fabricated by Yang et $\mathrm{al}^{8}$ at lower temperatures using pure methane precursor. The expanded view of the 2D peaks from samples grown at all four 
conditions are given in Figure S5. The FWHM of the 2D peak from the two-minute sample was lower $\left(\sim 53 \mathrm{~cm}^{-1}\right)$, indicative of a 3 layer system. The TEM image in Figure 1e confirms this conclusion. The very low thickness of the graphene film was further verified using UV-Vis spectroscopy. As evident from transmittance spectrum of the two-minute sample given in Figure S6, the films exhibited a transmittance of over $85 \%$ in the visible light region.

The peak in the Raman spectra observed at $\sim 1580 \mathrm{~cm}^{-1}(\mathrm{G})$ is believed to be originated from the doubly degenerate zone center phonon $\mathrm{E}_{2 \mathrm{~g}}$ mode and corresponds to the $\mathrm{C}-\mathrm{C}$ stretching mode ${ }^{24}$. In the samples fabricated for $1 \mathrm{~s}$ and $1 \mathrm{~min}$, the $\mathrm{G}$ peak was observed at $1584 \mathrm{~cm}^{-1}$, whereas it shifted to $1588 \mathrm{~cm}^{-1}$ in samples fabricated at longer processing time. The D peak observed at $1350 \mathrm{~cm}^{-1}$ is single and sharp, which is typical for graphene. For graphite, D peak is a band consisting of 2 peaks ${ }^{23}$. The $\mathrm{D}$ peak intensity was highest for the $1 \mathrm{~s}$ sample and lowest for 2 min sample. As the disorder of graphene increases the Raman intensity for the D peak (also $\mathrm{D}^{\prime}$ at $1620 \mathrm{~cm}^{-1}$ and $\mathrm{D}+\mathrm{G}$ at $2940 \mathrm{~cm}^{-1}$ ) increases ${ }^{25}$. The D peak arises from transverse optic phonon mode near K points in the Brillouin zone and represents scattering from intervalley defects. The SEM images (Figure 1a-d) showed that the effect of sheet boundary is highest for the $1 \mathrm{~s}$ sample. The images also showed the growth of folded graphene sheets and that the sheet boundary becomes increasingly more filled with the graphene sheets. However the 4 min sample shows a more folded structure that may contribute to a higher number of defects and a higher intensity of $\mathrm{D}$ peak. The type of disorder is not only defined by $\mathrm{sp}^{3}$ content but a different degree of $\mathrm{sp}^{2}$ clustering, which is affected by deposition conditions. The XPS spectra clearly show this influence in the sample.

The intravalley disorders give rise to the $\mathrm{D}$ peak at around $1620 \mathrm{~cm}^{-1}$. The $\mathrm{D}^{\prime}$ peak intensity is higher for vacancies than $\mathrm{sp}^{3}$ sites. $\mathrm{D}+\mathrm{G}$ peak $\left(2940 \mathrm{~cm}^{-1}\right)$ is a combination scattering peak. The Raman spectra in Figure 3 show D' peak at $1622 \mathrm{~cm}^{-1}$, with its intensity getting diminished as the duration of plasma discharge for synthesis increases which also increases the exposure of synthesized graphene to energetic plasma species. Thus, intravalley defects can be believed to be eliminated at the longer plasma exposure durations. Although small, $\mathrm{D}+\mathrm{G}$ and $\mathrm{G}+\mathrm{D}^{\prime}$ peaks were also observed at $2945 \mathrm{~cm}^{-1}$ and $3245 \mathrm{~cm}^{-1}$. 
The level of disorder in graphene and the number of layers can be assessed from $\mathrm{I}_{\mathrm{D}} / \mathrm{I}_{\mathrm{G}}$ and $\mathrm{I}_{2 \mathrm{D}} / \mathrm{I}_{\mathrm{G}}$ respectively, where ' $\mathrm{I}$ ' represents the intensity and these ratios are shown in Figure $3 \mathrm{~b}$. $T$ he $\mathrm{I}_{\mathrm{D}} / \mathrm{I}_{\mathrm{G}}$ is lowest (0.63) for 2 min sample, indicating that these samples have low defect density and are mostly composed of $\mathrm{sp}^{2}$ hybridized carbon. The $2 \mathrm{~min}$ sample has $\mathrm{I}_{2 \mathrm{D}} / \mathrm{I}_{\mathrm{G}}$ ratio highest (3.32) indicating that graphene with the minimum number of layers was grown at a plasma exposure time of 2 min ${ }^{23}$. $\mathrm{I}_{\mathrm{D}} / \mathrm{I}_{\mathrm{D}^{\prime}}$ above 13 is an indicator of graphene with higher $\mathrm{sp}^{3}$ defects, whereas a value around 7 implies vacancies and less than 5 the boundary defects. Only the samples prepared at duration up to 2 min exhibited the $\mathrm{D}^{\prime}$ peak. $\mathrm{I}_{\mathrm{D}} / \mathrm{I}_{\mathrm{D}^{\prime}}$ are 2.75 , 4.8 and 1.7 for $1 \mathrm{~s}, 1 \mathrm{~min}$ and 2 min samples respectively, indicating a higher contribution from boundary defects, which is also in agreement with the SEM results.

The properties exhibited by nanoscale materials, particularly those like graphene having atomic scale feature size, are often unpredictable and unique. Fabrication route has a critical role in deciding these properties as it controls surface/edge activities and charge carrier dynamics through manipulation of composition, structure and morphology. We found that the graphene grown from M. alternifolia extract was highly hydrophobic. Figure 4 shows the water-graphene contact angles for the 2 min sample on silicon substrate and for bare silicon. The graphene coated silicon yielded a contact angle of $135^{\circ}$ and hence, the surface is nearly superhydrophobic (contact angle $>150^{\circ}$ ). No significant change in contact angle was noticed in the case of $1 \mathrm{~s}$ samples or those fabricated on fused silica, which indicates that the contact angle is not controlled by the number of graphene layers or substrate. Rafiee et al reported that the graphene films grown on silicon substrates increased the contact angle of silicon by 1 to $2 \%$ only whereas those on glass increased the contact angle from $20^{\circ}$ (bare glass) to $94^{\circ}$ (graphene on glass) ${ }^{26}$. They also noticed no significant influence on contact angle from the number of graphene layers, especially when it is more than three. The contact angle that noticed in tea-tree graphene was very stable. To make sure that the high contact angle is not arising as a result of any adsorbed organic species, we subjected the films to high vacuum and also, sonication with acetone. While keeping in vacuum did not cause any change in the angle, acetone treatment reduced the contact angle to $120^{\circ}$. Nonetheless, the films regained original contact angle after several hours, which implies that the temporary reduction in contact angle was due to the adsorbed acetone. 
Planar graphene sheets are barely hydrophobic. Theoretical maximum for the water-graphene contact angle is about $96^{\circ}{ }^{27}$. Although a contact angle of $127^{\circ}$ was reported on planar graphene, it was considered unrealistic estimate by other groups ${ }^{28-30}$. Nevertheless, it is known that the wettability is influenced by chemical composition, geometric structure or both. A water-graphene contact angle of $160^{\circ}$ was reported by Rafiee et al in graphene films having a surface roughness of $10 \mu \mathrm{m}$ prepared by sonicating graphene flakes obtained by thermal exfoliation of graphite oxide in acetone and deposited on gold ${ }^{31}$. Superhydrophobicity was observed in graphene films with micron scale surface roughness and 3D graphene (or hybrid) foam network by other groups as well ${ }^{32-35}$. On the other hand, nanostructures of otherwise hydrophilic surfaces can also exhibit hydrophobic nature ${ }^{36}$. Yang et al reported a contact angle of $129^{0}$ for prestine graphene and $141^{0}$ for graphene grown on graphene oxide treated $\mathrm{SiO}_{2}$ substrates ${ }^{13}$. The transparency in their films dropped from $92 \%$ to $67 \%$ when the contact angle improved from $91^{\circ}$ to $129^{\circ}$. Nevertheless, the transmittance in our films that yielded a contact angle of $135^{\circ}$ was more than $85 \%$ (Figure S6). We believe that the very high hydrophobicity in our prestine graphene samples is primarily due to the nano-scale 3D architecture on the surface as evidenced by the AFM image in Figure S5. To the best of our knowledge this is the highest contact angle reported on graphene films having nanoscale feature size fabricated by a bottom up method.

Apart from water, we measured contact angles also of diiodomethane (non-polar), ethylene glycol (polar) and formamide (polar) on graphene (Figure S7). The values were used to determine surface energy of graphene using the van Oss-Chaudhury-Good (OCG) ${ }^{37}$ relation given by

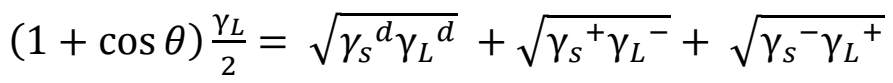

where $\theta$ and $\gamma$ are the contact angle and surface energy respectively. The subscripts $L$ and $S$ respectively represent the liquid and solid phases. The superscripts 'd', '+' and '-' represent respectively the dispersive and Lewis acid and base components of the surface tension. The values are tabulated in Table S1. The average surface energy obtained was $33 \mathrm{~mJ} / \mathrm{m}^{2}$, which is one of the lowest reported for graphene. While these results indicate that the material can be used for applications involving superhydrophobic surfaces, the low surface energy potentially gives stability to electronic devices developed using this graphene. 
The graphene films grown from $M$. alternifolia extract exhibited strong potential for use in resistive random access memory (RRAM) devices also. The RRAMs are considered next generation nonvolatile memory devices, which are also called memristors (memory resistance), that can be programmed in to a low resistance state (LRS) or a high resistance state (HRS) by the application of an external electric field ${ }^{38-40}$. A typical RRAM cell consists of a conductor/semiconductor (or insulator)/conductor sandwich configuration. Such a device formed by sandwiching a layer of the p-type organic semiconductor pentacene between graphene and aluminum electrodes (see Figure 5a) exhibited the memristive behavior. The I-V characteristics showed switching at $1.8 \mathrm{~V}$ (Figure 5b). No change in the magnitudes of the current corresponding to HRS and LRS states was observed when the device was subjected to over four hundred 'ON/OFF' cycles (Figures 5b, S8). Although graphene based electrodes were used for enhancing the performance of devices employing pentacene ${ }^{41,42}$ to the best of our knowledge, devices having strong memristive properties have not been reported. Memristive behavior was not observed when graphene was replaced by Gold or Silver. While the location of Fermi level in graphene relative to pentacene HOMO (highest occupied molecular orbital) level is believed to be primarily responsible for this behavior, it is likely that the low surface energy, as determined using contact angle measurements, also has a significant role. Further studies are underway to understand the actual role of the M. alternifolia based graphene in providing the memristive property to the graphene/pentacene/aluminum device.

In summary, we fabricated graphene films that possess a 3D nanostructured surface from natural M. alternifolia extract using a simple plasma assisted CVD process. The process is fast, sustainable, scalable and potentially low cost. The graphene composed primarily of $\mathrm{sp}^{2}$ hybridized carbon with traces of $\mathrm{sp}^{3}$ carbon and C-O bonds. The films were nearly defect free as confirmed by Raman studies. These graphene films were highly hydrophobic, which was attributed to the nano-scale surface features. The films are also proven to be highly useful for RRAM applications.

\section{Experimental Methods}

The deposition of graphene was carried out using plasma enhanced chemical vapor deposition (PECVD) in the absence of a catalyst. The experimental system consisted of a custom made 
quartz tube reactor, with a heater wound onto the quartz tube and controlled by a variable voltage controller. The base pressure of 0.05 mbar was initially obtained. $\mathrm{H}_{2}$ gas was flown at the rate of $30 \mathrm{sccm}$ and the system pressure is adjusted to be 0.20 mbar. The RF energy from the Navio RF generator (13.56 MHz) was delivered to the reactor via capacitive coupling through the Navio matching network. The system was optimized for an input RF energy of $500 \mathrm{~W}$ and temperature of $800{ }^{\circ} \mathrm{C}$. Cleaned Si wafers with $100 \mathrm{~nm}$ thermal oxide layer were used as substrates. Prior to deposition, Si samples were pretreated with plasma for 1 minute. M. alternifolia oil (99.9\% pure, Australian Botanical Products, Australia) was used as a carbon source. As M. alternifolia is volatile at ambient temperature, no heating was required to deliver the vapors from the flask into the chamber. The vapor flow rate was controlled using a flow controller.

Morphology and structural properties of M. alternifolia based graphene were investigated using confocal Raman spectroscopy (Rainshaw Raman Microscope, 514nm laser) and scanning electron microscopy (JOEL Field Emission SEM). Elemental composition of as-deposited graphene were characterized using x-ray photoelectron spectroscopy (AXIS-Hi, Shimadzu/KRATOS under basic pressure of $5.6 \times 10^{-9}$ Torr, X- Ray source used was anode mono-Al with pass energy of $40 \mathrm{eV}$ and $80 \mathrm{eV}$ ). $\mathrm{X}$-ray diffraction measurements were done using Rigaku Smartlab spectrometer at a glancing angle of $0.5^{\circ}$. The contact angles were measured using a home-made system involving an optical microscope (Leica).

Pentacene layer structure was deposited over the graphene fabricated on fused silica using vacuum evaporation technique, at a pressure of $10^{-5}$ Torr and deposition rate of $\sim 1 \mathrm{~nm} / \mathrm{min}$. The aluminum electrodes were also fabricated using the thermal evaporation technique ${ }^{43}$, 44 . DC voltage was applied using Keithley source meter 2400 to the graphene electrode with reference to the $\mathrm{Al}$ electrode and a ramp voltage with a sweeping rate of around $0.1 \mathrm{~V} / \mathrm{s}$ and the corresponding current was measured.

\section{ASSOCIATED CONTENT}

Supporting Information.

SEM $(1 \mu \mathrm{m})$ (Figure S1), optical image of graphene $12 \mathrm{~mm} \times 10 \mathrm{~mm}$ (Figure S2), AFM (Figure S3), High resolution XPS scans (Figure S4), Raman 2D peak (Figure S5), UV-Vis spectrum (Figure S6), contact angles (Figure S7) and on-off cycle of the mermistive device (Figure S8) are shown. This material is available free of charge via the Internet at http://pubs.acs.org. 


\section{Acknowledgement}

Authors are grateful to Ivy Ahiabu (XRD), University of Houston and P. Vivekanandan (XPS),

Toyo University for the help in material characterization. KB also acknowledges the ARC DECRA fellowship. Part of this research was supported by NIE/NTU AcRF grant (RI 7/11 RSR) provided by National Institute of Education, Nanyang Technological University, Singapore. OKV acknowledges the financial support from University of Houston.

\section{References}

1. Geim, A. K.; Novoselov, K. S. Nature materials 2007, 6, (3), 183-191.

2. Borunda, M. F.; Hennig, H.; Heller, E. J. Physical Review B 2013, 88, (12), 125415.

3. Hwang, E.; Sarma, S. D. Physical Review B 2008, 77, (11), 115449.

4. Hwang, E.; Sarma, S. D. Physical Review B 2013, 87, (11), 115432.

5. Novoselov, K. S.; Geim, A. K.; Morozov, S. V.; Jiang, D.; Zhang, Y.; Dubonos, S. V.; Grigorieva, I. V.; Firsov, A. A. Science 2004, 306, (5696), 666-669.

6. Seo, D. H.; Rider, A. E.; Han, Z. J.; Kumar, S.; Ostrikov, K. K. Advanced Materials 2013, 25, (39), 5638-5642.

7. Unarunotai, S.; Murata, Y.; Chialvo, C. E.; Mason, N.; Petrov, I.; Nuzzo, R. G.; Moore, J. S.; Rogers, J. A. Advanced Materials 2010, 22, (10), 1072-1077.

8. Yang, W.; Chen, G.; Shi, Z.; Liu, C.-C.; Zhang, L.; Xie, G.; Cheng, M.; Wang, D.; Yang, R.; Shi, D. Nature materials 2013, 12, (9), 792-797.

9. Peng, K.-J.; Wu, C.-L.; Lin, Y.-H.; Liu, Y.-J.; Tsai, D.-P.; Pai, Y.-H.; Lin, G.-R. Journal of Materials Chemistry C 2013, 1, (24), 3862-3870.

10. Kim, Y. S.; Joo, K.; Jerng, S.-K.; Lee, J. H.; Moon, D.; Kim, J.; Yoon, E.; Chun, S.-H. ACS Nano 2014, 8, (3), 2230-2236.

11. Zhang, L.; Shi, Z.; Wang, Y.; Yang, R.; Shi, D.; Zhang, G. Nano Res. 2011, 4, (3), 315-321.

12. Kim, H.; Son, Y.; Park, C.; Cho, J.; Choi, H. C. Angewandte Chemie International Edition 2013, 52, (23), 5997-6001.

13. Yang, C.; Bi, H.; Wan, D.; Huang, F.; Xie, X.; Jiang, M. Journal of Materials Chemistry A 2013, 1, (3), 770-775.

14. Li, Z.; Wu, P.; Wang, C.; Fan, X.; Zhang, W.; Zhai, X.; Zeng, C.; Li, Z.; Yang, J.; Hou, J. ACS nano 2011, 5, (4), 3385-3390.

15. Wang, J.; Wang, C.-F.; Chen, S. Angewandte Chemie International Edition 2012, 51, (37), 9297-9301.

16. Ruan, G.; Sun, Z.; Peng, Z.; Tour, J. M. ACS Nano 2011, 5, (9), 7601-7607.

17. Zhu, C.; Guo, S.; Fang, Y.; Dong, S. ACS Nano 2010, 4, (4), 2429-2437.

18. Sun, Z.; Yan, Z.; Yao, J.; Beitler, E.; Zhu, Y.; Tour, J. M. Nature 2010, 468, (7323), 549-552.

19. Anderson, L. J.; Jacob, M. V. Materials Science and Engineering B-Advanced Functional Solid-State Materials 2012, 177, (3), 311-315.

20. Bazaka, K.; Jacob, M. V.; Truong, V. K.; Wang, F.; Pushpamali, W. A. A.; Wang, J. Y.; Ellis, A. V.; Berndt, C. C.; Crawford, R. J.; Ivanova, E. P. Biomacromolecules 2010, 11, (8), 2016-2026.

21. Easton, C. D.; Jacob, M. V.; Shanks, R. A. Polymer 2009, 50, (15), 3465-3469.

22. Bo, Z.; Mao, S.; Han, Z. J.; Cen, K.; Chen, J.; Ostrikov, K. K. Chemical Society Reviews 2015. 
23. Ferrari, A.; Meyer, J.; Scardaci, V.; Casiraghi, C.; Lazzeri, M.; Mauri, F.; Piscanec, S.; Jiang, D.; Novoselov, K.; Roth, S. Physical review letters 2006, 97, (18), 187401.

24. Ferrari, A. C.; Basko, D. M. Nature nanotechnology 2013, 8, (4), 235-246.

25. Eckmann, A.; Felten, A.; Mishchenko, A.; Britnell, L.; Krupke, R.; Novoselov, K. S.; Casiraghi, C. Nano letters 2012, 12, (8), 3925-3930.

26. Rafiee, J.; Mi, X.; Gullapalli, H.; Thomas, A. V.; Yavari, F.; Shi, Y.; Ajayan, P. M.; Koratkar, N. A. Nat Mater 2012, 11, (3), 217-222.

27. Shih, C.-J.; Wang, Q. H.; Lin, S.; Park, K.-C.; Jin, Z.; Strano, M. S.; Blankschtein, D. Physical review letters 2012, 109, (17), 176101.

28. Wang, S.; Zhang, Y.; Abidi, N.; Cabrales, L. Langmuir 2009, 25, (18), 11078-11081.

29. Taherian, F.; Marcon, V.; van der Vegt, N. F.; Leroy, F. d. r. Langmuir 2013, 29, (5), 14571465.

30. Raj, R.; Maroo, S. C.; Wang, E. N. Nano Letters 2013, 13, (4), 1509-1515.

31. Rafiee, J.; Rafiee, M. A.; Yu, Z. Z.; Koratkar, N. Advanced Materials 2010, 22, (19), 21512154.

32. Liu, Y.; Zhang, J.; Li, S.; Wang, Y.; Han, Z.; Ren, L. RSC Advances 2014, 4, 45389 - 45396.

33. Dong, J.; Yao, Z.; Yang, T.; Jiang, L.; Shen, C. Scientific reports 2013, 3.

34. Singh, E.; Chen, Z.; Houshmand, F.; Ren, W.; Peles, Y.; Cheng, H. M.; Koratkar, N. Small 2013, 9, (1), 75-80.

35. Dong, X.; Chen, J.; Ma, Y.; Wang, J.; Chan-Park, M. B.; Liu, X.; Wang, L.; Huang, W.; Chen, P. Chemical Communications 2012, 48, (86), 10660-10662.

36. Feng, L.; Li, S.; Li, Y.; Li, H.; Zhang, L.; Zhai, J.; Song, Y.; Liu, B.; Jiang, L.; Zhu, D. Advanced materials 2002, 14, (24), 1857-1860.

37. Van Oss, C.; Chaudhury, M.; Good, R. Advances in colloid and interface science 1987, 28, 35-64.

38. Liu, G.; Zhuang, X.; Chen, Y.; Zhang, B.; Zhu, J.; Zhu, C.-X.; Neoh, K.-G.; Kang, E.-T. Applied Physics Letters 2009, 95, (25), -.

39. Son, J. Y.; Shin, Y.-H.; Kim, H.; Jang, H. M. ACS Nano 2010, 4, (5), 2655-2658.

40. Wang, L.-H.; Yang, W.; Sun, Q.-Q.; Zhou, P.; Lu, H.-L.; Ding, S.-J.; Zhang, D. W. Applied Physics Letters 2012, 100, (6), 063509.

41. Basu, S.; Lee, M. C.; Wang, Y.-H. Physical Chemistry Chemical Physics 2014, 16, (31), 16701-16710.

42. Berke, K.; Tongay, S.; McCarthy, M.; Rinzler, A.; Appleton, B.; Hebard, A. Journal of Physics: Condensed Matter 2012, 24, (25), 255802.

43. Taguchi, D.; Manaka, T.; Iwamoto, M.; Anderson, L. J.; Jacob, M. V. Chemical Physics Letters 2014.

44. Taguchi, D.; Manaka, T.; Iwamoto, M.; Bazaka, K.; Jacob, M. V. Chemical Physics Letters 2013, 572, 150-153. 


\section{Figures}
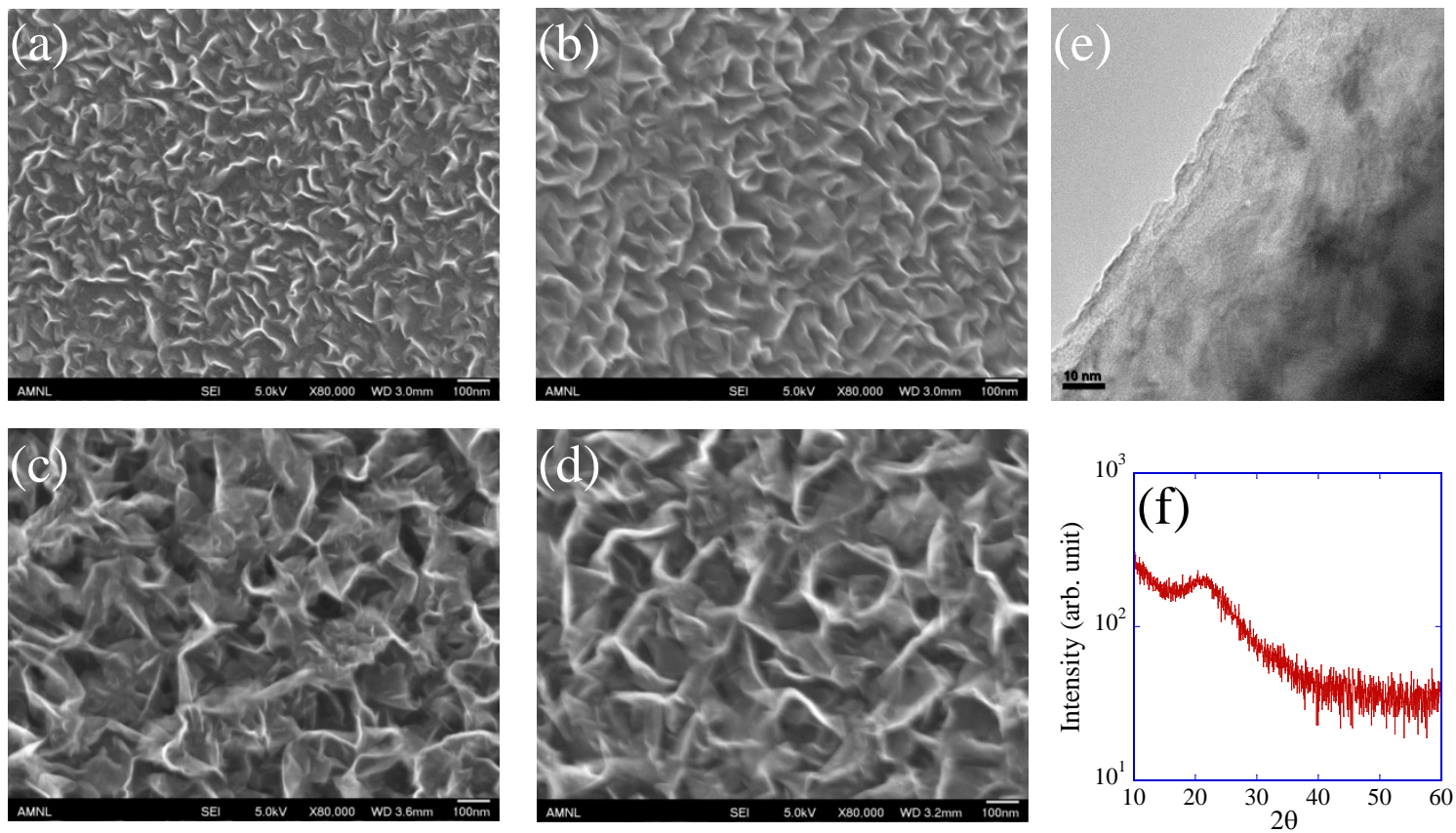

Figure 1: SEM images of samples fabricated on $1 \mathrm{~cm} \times 1 \mathrm{~cm}$ silicon substrates for $1 \mathrm{~s} \mathrm{(a),} 1 \mathrm{~min}$ (b), 2 min (c) and 4 min (d), TEM image of a 2 min graphene sample (e) and GAXRD pattern obtained from a 2 min sample (f). 

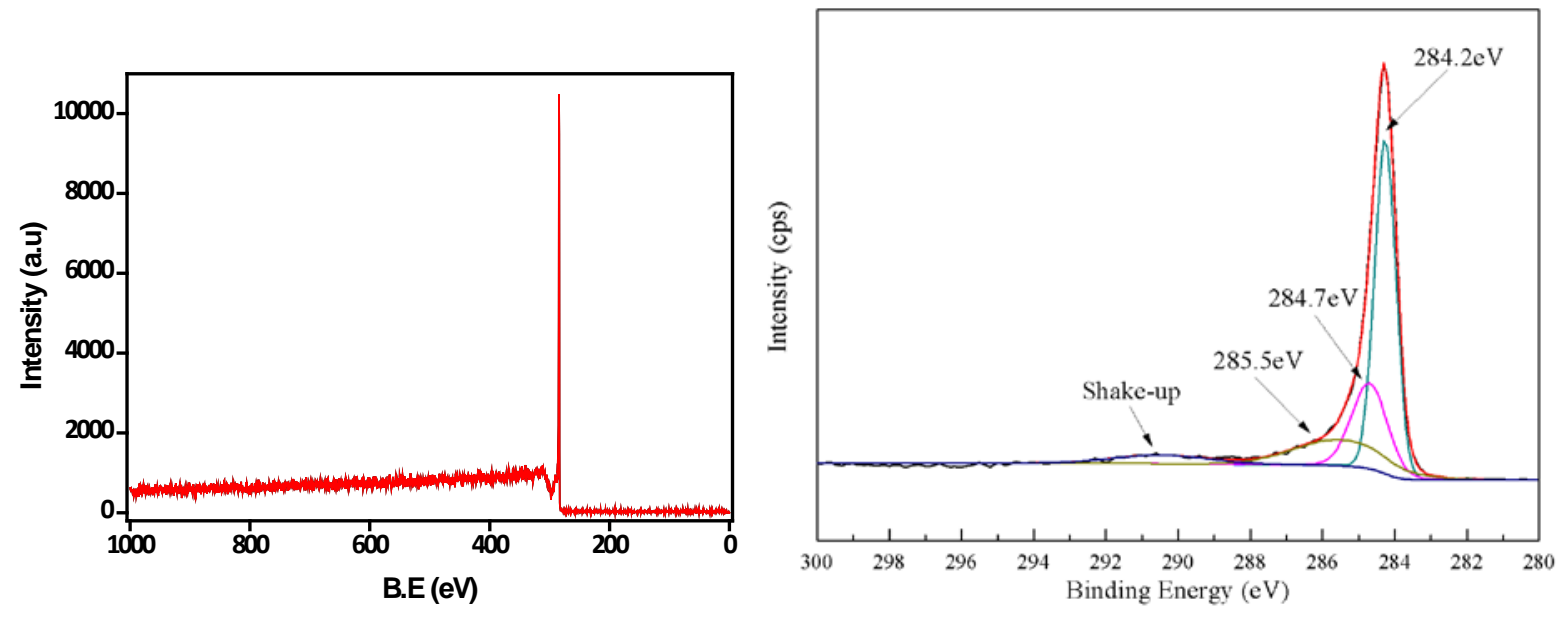

Figure 2 XPS spectra of the 2 min graphene survey Scan (a), high resolution (b). 

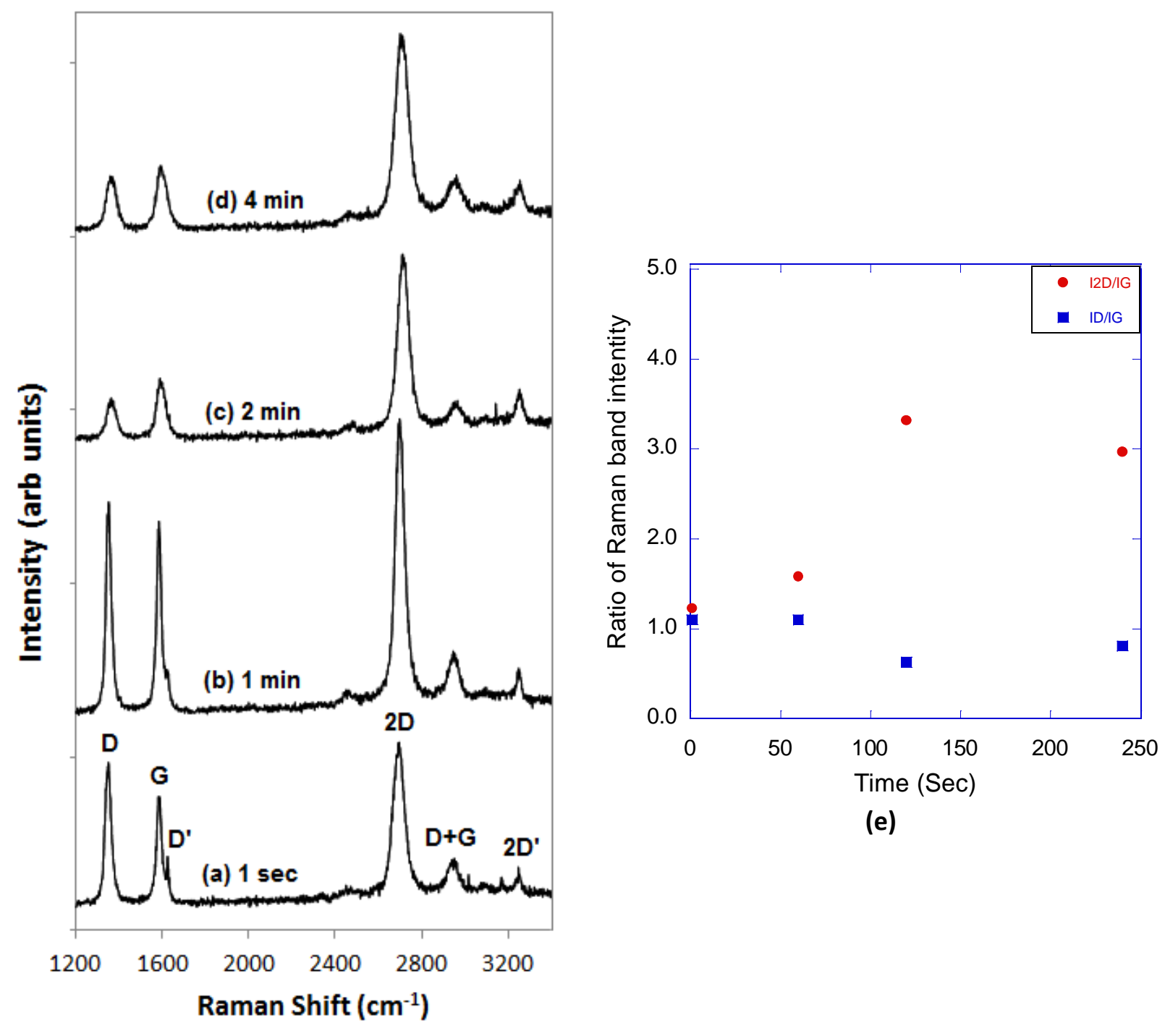

(e)

Figure 3: (a) Raman spectra of graphene samples deposited for $1 \mathrm{~s}, 1 \mathrm{~min}, 2 \mathrm{~min}$ and $4 \mathrm{~min}$ and (b) the ratio of intensity peaks for different graphene samples (e). 


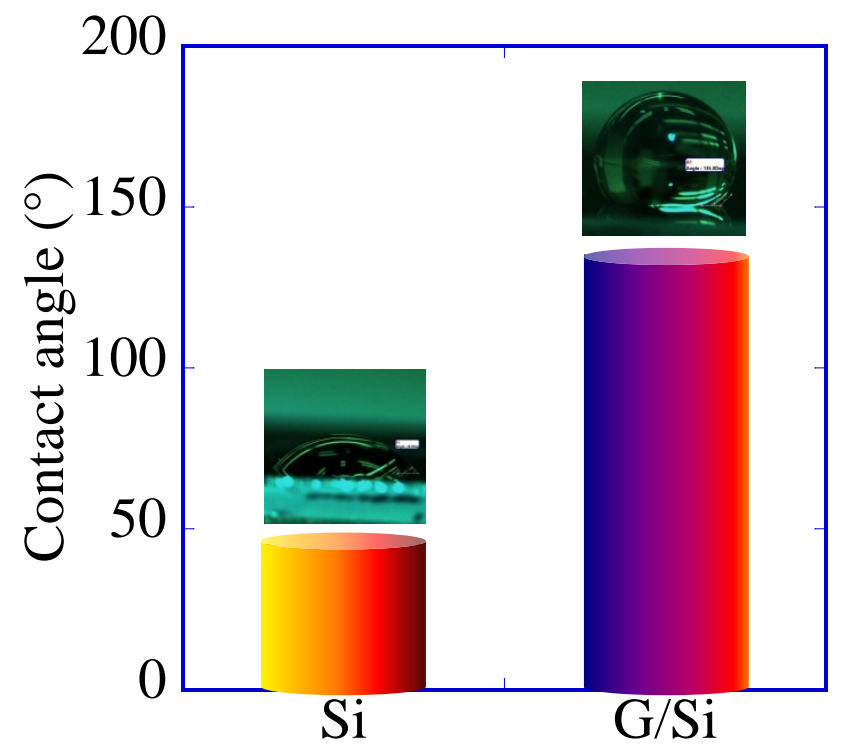

Figure 4: Contact angles of water on the surface of bare silicon (Si) and 2 min graphene coated silicon (G/Si). 
a)
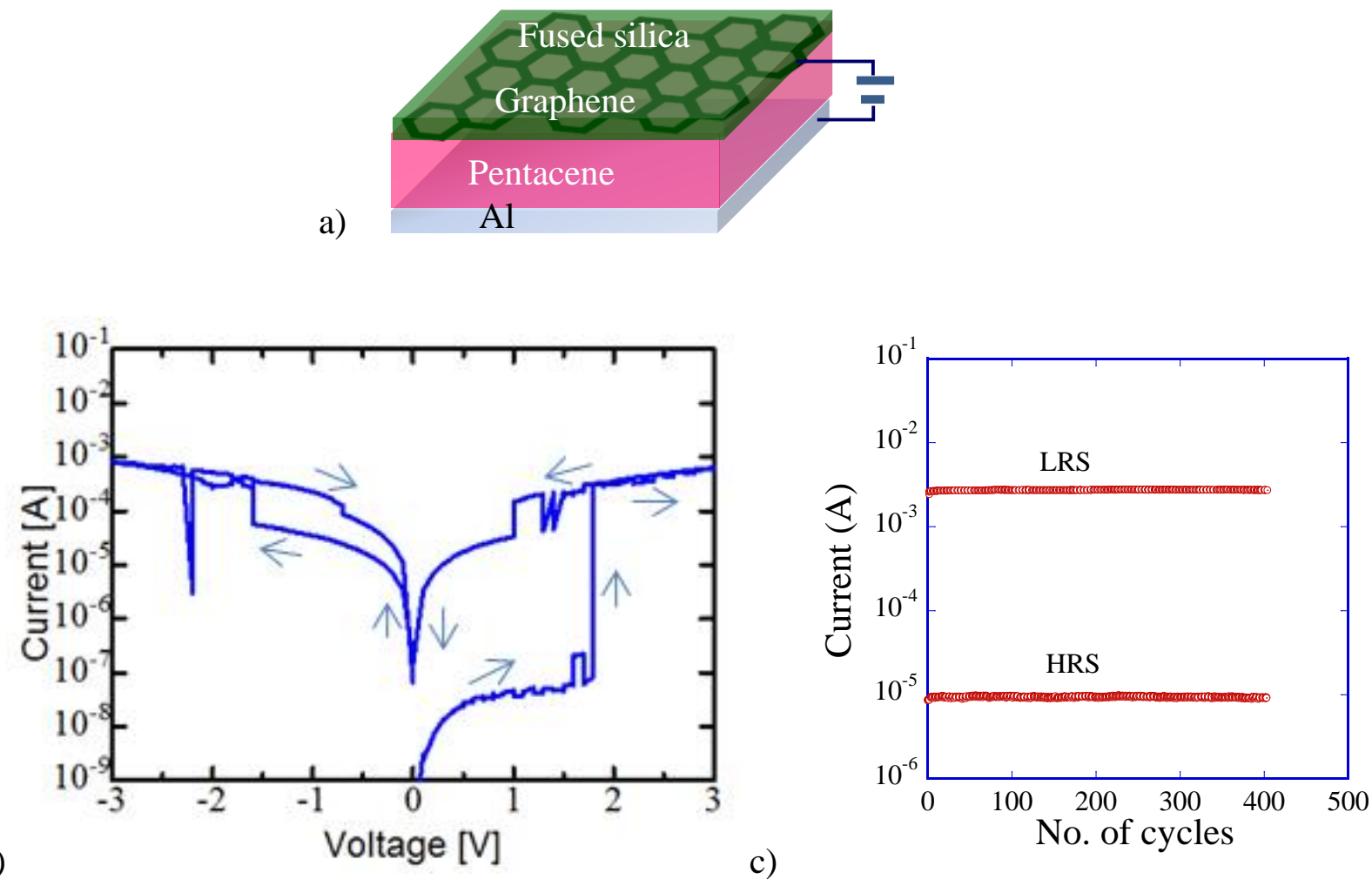

b)

c)

Figure 5: Memory device configuration (a), I-V Characteristics (b) and the retention plot showing HRS and LRS over 400 cycles taken over 10 minutes (c) 